\title{
Alteration of $\mathrm{CD} 4 \mathrm{~T}$ cell subsets in metastatic lymph nodes of human gastric cancer
}

\author{
YOSHIHIRO OKITA, MASAICHI OHIRA, HIROAKI TANAKA, MAO TOKUMOTO, YUKIE GO, \\ KATSUNOBU SAKURAI, TAKAHIRO TOYOKAWA, NAOSHI KUBO, KAZUYA MUGURUMA, \\ TETSUJI SAWADA, KIYOSHI MAEDA and KOSEI HIRAKAWA
}

Department of Surgical Oncology, Osaka City University Graduate School of Medicine, Abeno-ku, Osaka 545-8585, Japan

Received April 29, 2015; Accepted June 2, 2015

DOI: $10.3892 /$ or.2015.4064

\begin{abstract}
Studies of tumor-infiltrating immune cells have revealed that immune escape plays an important role in tumor growth. The aim of the present study was to investigate the impact of metastasis affecting $\mathrm{CD}^{+} \mathrm{T}$ cell subsets in human clinical samples. Single-cell suspensions derived from tumor-draining lymph node (TDLN) and primary cancer specimens were assessed by flow cytometry, qRT-PCR and immunohistochemistry. In the $\mathrm{CD}^{+} \mathrm{T}$ cell subsets detected in TDLN, effector T cells $\left(\mathrm{T}_{\mathrm{E}}\right)$ in metastatic TDLN (mTDLN) was significantly lower than that in metastatic-free TDLN $(\mathrm{mfTDLN}) . \mathrm{T}_{\mathrm{E}}$ in mfTDLN were increased compared with normal controls. Similarly, effector memory $T$ cells $\left(\mathrm{T}_{\mathrm{EM}}\right)$ in mTDLN was significantly lower than in control and mfTDLN. There was a significantly positive correlation between the proportion of $\mathrm{T}_{\mathrm{EM}}$ in TDLN and number of tumor-infiltrating $\mathrm{CD}^{+}$and $\mathrm{CD}^{+} \mathrm{T}$ cells. Th1 to $\mathrm{Th} 2$ ratio was lower in mTDLN, and Treg in mTDLN was significantly higher than in mfTDLN. CD4 ${ }^{+} \mathrm{T}$ cell and $\mathrm{T}_{\mathrm{E}}$ subsets in TDLN were significantly affected by metastasis. Immunosuppressive cells exhibit increased migration to TDLN, in which a subset of $\mathrm{CD}^{+} \mathrm{T}_{\mathrm{E}}$ is skewed towards immune tolerance in the tumor microenvironment.
\end{abstract}

\section{Introduction}

The immune system functions as a host defensive mechanism protecting against tumor development. Evidence suggests that cell autoantigens, which are displayed by antigen-presenting cells (APC) on MHC class II molecules, stimulate $\mathrm{CD}^{+} \mathrm{T}$ helper cells in local tumor-draining lymph node (TDLN) (1).

Correspondence to: Dr Hiroaki Tanaka, Department of Surgical Oncology, Osaka City University Graduate School of Medicine, 1-4-3 Asahimachi, Abeno-ku, Osaka 545-8585, Japan

E-mail: hiroakitan@med.osaka-cu.ac.jp

Key words: CD4 T cells, gastric cancer, lymph node metastasis, effector T cell subsets, $\mathrm{T}$ cell subsets, flow cytometry, immunology
Although numerous antigen-specific $\mathrm{T}$ lymphocytes proliferate in draining lymph nodes, advanced-stage cancer cells are able to invade TDLN from primary lesions. Thus, it is important to investigate the relationship between lymph node metastasis and the underlying mechanisms of immune tolerance in TDLN. However, few studies have examined changes in immune cells in lymph nodes of human clinical samples.

T lymphocytes are divided into three subsets: naïve $\left(\mathrm{T}_{\mathrm{N}}, \mathrm{CD} 45 \mathrm{RA}^{+} \mathrm{CCR} 7^{+}\right)$, effector $\left(\mathrm{T}_{\mathrm{E}}, \mathrm{CD} 45 \mathrm{RA}^{+} \mathrm{CCR} 7^{-}\right)$and memory $\mathrm{T}$ cells (CD45RA ${ }^{-}$). Memory $\mathrm{T}$ cells are further divided into effector memory $\mathrm{T}$ cells ( $\mathrm{T}_{\mathrm{EM}}, \mathrm{CD}^{2} \mathrm{RA}^{-} \mathrm{CCR} 7^{-}$), which are short-lived tissue-resident memory cells and central memory $\mathrm{T}$ cells $\left(\mathrm{T}_{\mathrm{CM}}, \mathrm{CD} 45 \mathrm{RA}^{-} \mathrm{CCR} 7^{+}\right)$, which are long-lived memory cells that are found in secondary lymphoid organs $(2,3)$. After an immune response is induced, antigen-responsive $\mathrm{T}_{\mathrm{CM}}$ and $\mathrm{T}_{\mathrm{EM}}$ persist in the memory pool and provide systemic immune surveillance in lymphoid organs. In turn, a previous study revealed that $\mathrm{CD}^{+} \mathrm{T}_{\mathrm{EM}}$ in cervical cancer metastatic TDLN were fewer than in metastasis free-TDLN (4).

Previous studies investigating tumor-infiltrating effector $\mathrm{CD}^{+} \mathrm{T}$ cells revealed that immune escape plays an important role in tumor growth and the incidence of cancer. Notably, a high Th1 to Th2 ratio among tumor-infiltrating lymphocytes is positively correlated with better prognosis in various types of carcinoma (5-8). The suppression of antitumor $\mathrm{T}$ cell responses leads to increased regulatory T cells (Treg) in TIL and TDLN and is correlated with poor prognosis (9-15). We previously

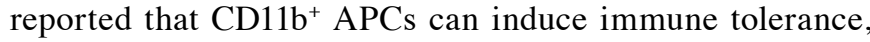

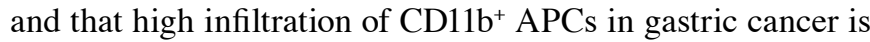
associated with poor prognosis (16). Recently, the increased infiltration of tumor-associated neutrophils (TAN) in primary tumors has been shown to correlate with poor prognosis in head and neck, and gastric cancers (17-19).

In mice, several studies have examined the role of T-cell tolerance and tumor-infiltrating cells in the cancer microenvironment. However, the cancer progression-dependent changes of immune cells in TDLN in humans remain unclear. Thus, the aim of the present study was to investigate the impact of metastasis and other $\mathrm{X}$ factors on the $\mathrm{CD} 4^{+} \mathrm{T}$ cell subset in human clinical samples of TDLN to shed light on cellular immune regulation in tumor microenvironments related to gastric cancer. 


\section{Materials and methods}

Clinical samples. TDLN and primary tumors were collected from 28 consecutive patients who underwent surgical resection for gastric adenocarcinoma at the Osaka City University Graduate School of Medicine between 2011 and 2013. Pathological stage was determined according to the Japanese classification of gastric carcinoma: 3rd edition (20). The present study was approved by the Medical Ethics Committee of Osaka City University Hospital and written informed consent was obtained from each patient before treatment.

We obtained about five D1 regional lymph node samples per one case according to the Japanese gastric cancer treatment guidelines 2010 (ver. 3) (21). Half of the lymph nodes were used for making single cell suspensions, remaining lymph nodes were determined for possible lymph node metastasis by immunohistochemistry. Since we thought that necrotic changes skew immune profiles in TDLN, lymph nodes with macroscopic metastasis were excluded in the present study. Fresh lymph node samples were suspended in $5 \mathrm{ml}$ Roswell Park Memorial Institute (RPMI)-1640 complete medium (Wako, Osaka, Japan) supplemented with $10 \%$ fetal bovine serum (FBS; Nichirei Biosciences, Tokyo, Japan) and $2 \%$ penicillin/streptomycin (Wako). The lymph node samples were washed twice with phosphate-buffered saline (PBS), sliced with a scalpel into $\sim 2-3 \mathrm{~mm}^{3}$ pieces and then passed through a cell strainer (Becton-Dickinson, San Diego, CA, USA). The obtained single-cell suspensions were mixed with $900 \mu 1 \mathrm{FBS}$ and $100 \mu 1$ dimethylsulfoxide (Wako), and were then cryopreserved at $-80^{\circ} \mathrm{C}$ until needed for analysis. After pathological diagnosis, we divided TDLN into three groups: control, metastasis-free TDLN (mfTDLN) and metastatic TDLN (mTDLN). We defined the control as TDLN from patients with no lymph node metastasis who underwent surgical resection for gastric cancer. Both mfTDLN and mTDLN were obtained from the same patient. We applied and compared among one control, one mfTDLN and one mTDLN per patient. The single-cell suspensions were separated into CD4-positive and -negative cells using a Dynal CD4 Positive Isolation kit (Life Technologies, Carlsbad, CA, USA) according to the manufacturer's instructions.

Phenotypic analysis.CD4-positive single-cell suspensions were analyzed by polychromatic flow cytometry. Briefly, the cells were incubated with mouse anti-human CD3-APC, CD4-FITC, CD45RA-PECy7, CCR7-PE, CD25-PECy7 or programmed cell death-1 (PD-1)-PE antibodies (BD Pharmingen, San Diego, CA, USA). To detect myeloid-derived APCs and neutrophils, whole single-cell suspensions collected from lymph nodes were incubated with CD11b-PE, CD11c-APC, HLA-DR-FITC, programmed cell death ligand 1 (PD-L1)-PE or CD16b-PE antibodies (BD Pharmingen), and then analyzed by flow cytometry. Flow cytometric analyses were performed using a BD LSR II flow cytometer with FACSDiva ${ }^{\mathrm{TM}}$ software (both from Becton-Dickinson).

Immunohistochemistry. Primary tumor specimens of the largest size from each patient were fixed in $10 \%$ formalin, embedded in paraffin and serially sectioned (4- $\mu$ m thickness) for immunohistochemistry analysis. After antigen retrieval by autoclaving at $105^{\circ} \mathrm{C}$ for $10 \mathrm{~min}$, the tissue sections were incubated overnight at $4^{\circ} \mathrm{C}$ with rabbit anti-human anti-CD4 monoclonal antibody (ab133616, 1:100 dilution; Abcam, Cambridge, UK) or anti-CD8 monoclonal antibody (NCL-CD8-4B11, 1:40 dilution; Leica, Newcastle, UK). The signal was amplified by streptavidin-biotin complex formation and developed with 3,3'-diaminobenzidine (DAB) followed by counterstaining with hematoxylin. All cell counts were performed using a BX41 microscope (Olympus, Tokyo, Japan) at x200 magnification (x20 objective and x10 eyepiece). Samples were scored in a blinded manner by two investigators with respect to clinicopathological features. For each section, five areas of a representative field of tumor were assessed.

Intracellular staining and T cell activation. Intracellular staining for FOXP3 (Ab-PE) was performed using a FOXP3 buffer set (BD Pharmingen) according to the manufacturer's instructions. To assess the $\mathrm{T}_{\mathrm{E}}$ subset, isolated $\mathrm{CD} 4^{+} \mathrm{T}$ cells were activated using a Leukocyte Activation Cocktail with GolgiPlug $^{\mathrm{TM}}$ (BD Pharmingen) according to the manufacturer's instructions. Briefly, lymph node lymphocytes were stimulated with the Leukocyte Activation Cocktail mixture containing a phorbol ester (phorbol 12-myristate 13-acetate, PMA), calcium ionophore (Ionomycin), and protein transport inhibitor (BD GolgiPlug ${ }^{\mathrm{TM}}$, containing brefeldin A) in 24-well plates at a concentration of $1 \times 10^{6}$ cells $/ \mathrm{ml}$ for $5 \mathrm{~h}$ at $37^{\circ} \mathrm{C}$ with $5 \% \mathrm{CO}_{2}$ in RPMI-1640 complete medium. To stain the cell surface, the activated cells were mixed with mouse anti-human FITC-conjugated anti-CD4 antibody, and incubated for $30 \mathrm{~min}$ on ice. To detect intracellular cytokines, a Cytofix/Cytoperm intracellular cytokine staining kit (BD Pharmingen) was used according to the manufacturer's instructions. Briefly, cells were re-suspended in $100 \mu \mathrm{l}$ Cytofix/Cytoperm ${ }^{\mathrm{TM}}$ solution for $20 \mathrm{~min}$ at $4^{\circ} \mathrm{C}$ for fixation and permeabilization, and were then washed twice in $1 \mathrm{X}$ $\mathrm{Perm} / \mathrm{Wash}^{\mathrm{TM}}$ solution, pelleted by centrifugation at $400 \mathrm{xg}$ for $5 \mathrm{~min}$ at $4^{\circ} \mathrm{C}$, and separated from the supernatant. After fixation and permeabilization, cells were incubated with appropriate antibodies for $30 \mathrm{~min}$ on ice.

Quantitative real-time $(R T)-P C R$. Total RNA was prepared using an RNeasy Micro kit (Qiagen, Chatsworth, CA, USA) according to the manufacturer's instructions. cDNA was synthesized from $100 \mathrm{ng}$ mRNA by standard RT-PCR amplification with ReverTra Ace qPCR RT Master mix (Toyobo, Osaka, Japan). TaqMan PCRs for interferon (IFN)- $\gamma$ (Hs00989291_m1), interleukin (IL)12A (IL-12p35, Hs01073447_m1), IL-8 (Hs00174103_m1), matrix metalloproteinase 2 (MMP2) (Hs01548727_m1) and matrix metalloproteinase 9 (MMP9) (Hs00234579_m1) were performed using an ABI Prism Detection System (Applied Biosystems). For amplification, $2.5 \mu \mathrm{l}$ cDNA was incubated with $12.5 \mu \mathrm{l} 2 \mathrm{X}$ TaqMan Master mix (8\% glycerol, $1 \mathrm{X}$ TaqMan buffer, $200 \mu \mathrm{mol} / 1 \mathrm{dATP}, 200 \mu \mathrm{mol} / 1 \mathrm{dGTP}$, $200 \mu \mathrm{mol} / 1 \mathrm{dCTP}, 400 \mu \mathrm{mol} / 1 \mathrm{dUTP}, 0.05 \mathrm{U} / \mu \mathrm{l}$ AmpErase uracil $\mathrm{N}$-glycosylase, $5 \mathrm{mmol} / \mathrm{l} \mathrm{MgCl}_{2}$ and $0.01 \mathrm{U} / \mu \mathrm{l}$ AmpliTaq Gold; Perkin-Elmer, Wellesley, MA, USA), $2.5 \mu 1$ TaqMan probe and sense and reverse primers at a final concentration of $10 \mathrm{pmol} / \mu 1$. The reaction mixture was adjusted to a final volume of $25 \mu 1$ with RNase-free distilled water. For quantification of 
Table I. Correlation between lymph node metastasis and clinicopathological characteristics in gastric cancer.

\begin{tabular}{|c|c|c|c|c|c|c|}
\hline \multirow[b]{2}{*}{ Variables } & \multirow[b]{2}{*}{$\mathrm{N}$} & \multicolumn{2}{|c|}{ Lymph node status } & \multirow[b]{2}{*}{ P-value } & \multirow[b]{2}{*}{ HR } & \multirow[b]{2}{*}{$95 \% \mathrm{CI}$} \\
\hline & & $\begin{array}{l}\text { Control } \\
(\mathrm{n}=13)\end{array}$ & $\begin{array}{l}\text { Metastatic LN } \\
\quad(\mathrm{n}=15)\end{array}$ & & & \\
\hline \multicolumn{7}{|l|}{ Gender } \\
\hline Male & 20 & 9 & 11 & 0.453 & 2.065 & $0.298-14.261$ \\
\hline Female & 8 & 4 & 4 & & & \\
\hline Age (years) & & 68 & 67 & 0.789 & 0.990 & $0.918-1.066$ \\
\hline \multicolumn{7}{|l|}{ Histological type } \\
\hline Intestinal & 12 & 4 & 8 & 0.226 & 2.571 & $0.562-13.207$ \\
\hline Diffuse & 16 & 9 & 7 & & & \\
\hline Tumor size (mm) & & 49.5 & 62.2 & 0.257 & 0.985 & $0.955-1.010$ \\
\hline \multicolumn{7}{|l|}{$\mathrm{T}$} \\
\hline $1-2$ & 21 & 13 & 8 & 0.705 & 0.750 & $0.169-3.325$ \\
\hline $3-4$ & 7 & 0 & 7 & & & \\
\hline \multicolumn{7}{|l|}{$\mathrm{V}$} \\
\hline$(-)$ & 23 & 11 & 12 & 0.824 & 0.8 & $0.091-5.776$ \\
\hline$(+)$ & 5 & 2 & 3 & & & \\
\hline \multicolumn{7}{|l|}{ Ly } \\
\hline$(-)$ & 11 & 6 & 5 & 0.488 & 0.583 & $0.121-2.687$ \\
\hline$(+)$ & 17 & 7 & 10 & & & \\
\hline \multicolumn{7}{|l|}{ Stage } \\
\hline 1 & 9 & 7 & 2 & 0.007 & 7.512 & $1.727-32.623$ \\
\hline 2 & 10 & 6 & 4 & & & \\
\hline 3 & 7 & 0 & 7 & & & \\
\hline 4 & 2 & 0 & 2 & & & \\
\hline
\end{tabular}

CI, confidence interval; HR, hazard ratio; Ly, lymphovascular invasion.

the human housekeeping gene glyceraldehyde-3-phosphate dehydrogenase, a Pre-Developed Assay kit (Perkin-Elmer) was used. The amplification was performed and analyzed using an ABI Prism 7700 Sequence Detector. To rule out contamination from buffers and tubes, a negative control with water in place of the cDNA template was included on every plate.

Statistical analysis. The association between lymph node metastasis and various clinicopathological variables was examined, and the significance of different prognostic markers was evaluated using binomial logistic regression analysis. The level of significance was set at $\mathrm{P}<0.05$. Statistical analyses were performed using SPSS 13.0 statistical software (SPSS, Inc., Chicago, IL, USA).

\section{Results}

Impact of metastasis on differentiation of $C D 4^{+} T$ cells in lymph nodes. Fresh lymph node samples were collected from 28 gastric adenocarcinoma patients, which consisted of 15 patients with pathological nodal metastasis and 13 patients without metastasis. No significant differences between patient characteristics, including gender, age, histological type, tumor size and lymphovascular invasion were detected based on lymph node metastasis status (Table I). Flow cytometric analysis revealed that the purity of isolated $\mathrm{CD}^{+} \mathrm{T}$ cells was $>98 \%$ (data not shown).

To examine the inhibitory effect of metastasis on $\mathrm{CD}^{+} \mathrm{T}$ cell differentiation in TDLN, we investigated the proportion of four subsets of $\mathrm{CD}^{+} \mathrm{T}$ cells: $\mathrm{T}_{\mathrm{E}}, \mathrm{T}_{\mathrm{N}}, \mathrm{T}_{\mathrm{EM}}$ and $\mathrm{T}_{\mathrm{CM}}$, and calculated the percentage of each group among total $\mathrm{CD}^{+} \mathrm{T}$ cells isolated from the patients lymph nodes based on flow cytometric analysis. As shown in Fig. 1A and B, the percentage of $T_{E}$ cells in mTDLN was significantly lower than that in mfTDLN $(\mathrm{P}=0.021) . \mathrm{T}_{\mathrm{E}}$ cells in mfTDLN were also increased compared with the normal control samples, whereas the number of $\mathrm{T}_{\mathrm{EM}}$ cells in $\mathrm{mTDLN}$ was significantly lower than in the control samples $(\mathrm{P}=0.010)$ and mfTDLN $(\mathrm{P}=0.041)$. No significant differences in the proportions of $\mathrm{T}_{\mathrm{N}}$ and $\mathrm{T}_{\mathrm{CM}}$ cells were detected. Despite the lack of CCR7, the lymph node homing receptor, in TDLN, $\mathrm{T}_{\mathrm{EM}}$ were the predominant $\mathrm{CD}^{+} \mathrm{T}$ cell subset in TDLN.

We next examined the correlation between the number of tumor-infiltrating $\mathrm{CD}^{+}$and $\mathrm{CD}^{+} \mathrm{T}$ cells in primary tumors and $\mathrm{CD} 4^{+} \mathrm{T}$ cell subset in TDLN. As shown in Fig. $1 \mathrm{C}$ and D, 

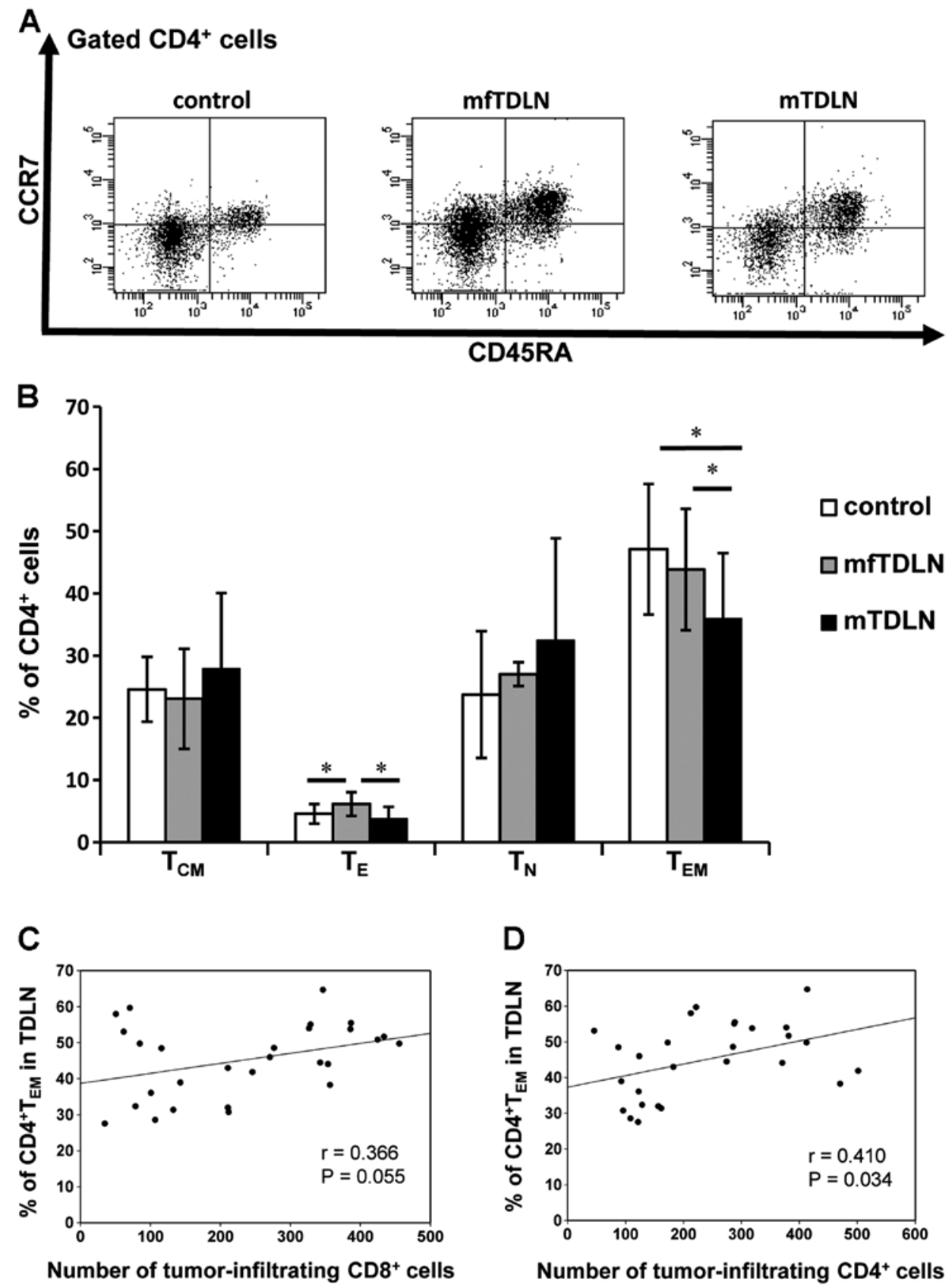

Figure 1. Flow cytometric analysis of $\mathrm{CD}^{+} \mathrm{T}$ cell subsets in TDLN. (A) Cells in TDLN were mechanically dissociated into single-cell suspensions and $\mathrm{CD} 4^{+} \mathrm{T}$ cells were then obtained using a CD4 ${ }^{+} \mathrm{T}$ cell isolation kit in combination with flow cytometry. Results were gated on $\mathrm{CD} 4^{+}$cells, and representative dot plots of control TDLN, mfTDLN and mTDLN are shown. $\mathrm{CD}^{+}$cells were subdivided into four populations: $\mathrm{CD}^{2} 5 \mathrm{RA}^{-} \mathrm{CCR} 7^{-}$cells $\left(\mathrm{T}_{\mathrm{EM}}\right)$, $\mathrm{CD}^{-} \mathrm{CRA}^{-} \mathrm{CCR} 7^{+}$ cells $\left(\mathrm{T}_{\mathrm{CM}}\right), \mathrm{CD}^{2} 5 \mathrm{RA}^{+} \mathrm{CCR} 7^{-}$cells $\left(\mathrm{T}_{\mathrm{E}}\right)$ and $\mathrm{CD} 45 \mathrm{RA}^{+} \mathrm{CCR} 7^{+}$cells $\left(\mathrm{T}_{\mathrm{N}}\right)$. (B) Proportion of each $\mathrm{T}$ cell subset among total $\mathrm{CD} 4^{+} \mathrm{T}$ cells was compared between control TDLN (white bars), mfTDLN (gray bars) and mTDLN (black bars). Histograms show mean values \pm SD from analyses performed for 13 control TDLN, $15 \mathrm{mfTDLN}$ and $15 \mathrm{mTDLN}$. Comparisons between groups were performed using the t-test. The number of $\mathrm{T}_{\mathrm{E}}$ cells in mfTDLN was significantly higher than in control $(\mathrm{P}=0.025)$ and mTDLN $(\mathrm{P}=0.021) . \mathrm{T}_{\mathrm{EM}}$ in mTDLN was significantly lower than in control and mfTDLN. No significant differences in $\mathrm{T}_{\mathrm{N}}$ or $\mathrm{T}_{\mathrm{CM}}$ were detected. (C and D) Spearman's correlation coefficients were used to determine the relationship between the proportion of CD4 ${ }^{+} \mathrm{T}_{\mathrm{EM}}$ cells in control and mTDLN and the number of tumor-infiltrating (C) $\mathrm{CD} 4^{+}$or (D) $\mathrm{CD} 8^{+} \mathrm{T}$ cells. Tumor-infiltrating $\mathrm{CD} 4^{+} \mathrm{T}$ cells had a significantly positive correlation with $\mathrm{CD}^{+} \mathrm{T}_{\mathrm{EM}}$ in TDLN ( $\left.\mathrm{r}=0.410, \mathrm{P}=0.034\right)$. Tumor-infiltrating $\mathrm{CD} 8^{+} \mathrm{T}$ cells were correlated with $\mathrm{CD} 4^{+} \mathrm{T}_{\mathrm{EM}}$ in $\mathrm{TDLN}$, yet the association was not significant $(\mathrm{r}=0.366, \mathrm{P}=0.055)$. Asterisk $(*)$ shows a significant difference $(\mathrm{P}<0.05)$. TDLN, tumor-draining lymph node; mfTDLN, metastatic-free TDLN; mTDLN, metastatic TDLN; $\mathrm{T}_{\mathrm{EM}}$, effector memory $\mathrm{T}$ cells; $\mathrm{T}_{\mathrm{E}}$, effector $\mathrm{T}$ cells.

a positive relationship was observed for $\mathrm{CD}^{+}$and $\mathrm{T}_{\mathrm{EM}}$ cells $(\mathrm{r}=0.410, \mathrm{P}=0.034)$, suggesting that $\mathrm{T}_{\mathrm{EM}}$ cells in TDLN could be considered tumor-related $\mathrm{CD} 4^{+} \mathrm{T}$ cells.

Impact of metastasis on effector T cell subsets in lymph nodes. To investigate the effects of metastasis on the function of effector $\mathrm{T}$ cell subsets, we examined differences in the proportions of $\mathrm{T}_{\mathrm{E}}$
$\mathrm{CD} 4^{+}$cells among normal control lymph nodes, mfTDLN and mTDLN by intracellular cytokine detection using multicolor flow cytometric analysis. The examined cell populations were defined as follows: Th1, INF- $\gamma$-positive and IL-4-negative; Th2, INF- $\gamma$-negative and IL-4-positive; Th17, IL-17-positive; and Treg, CD25-positive and FOXP3-positive. As shown in Fig. 2A, the Th1/Th2 ratio significantly decreased in both 
A
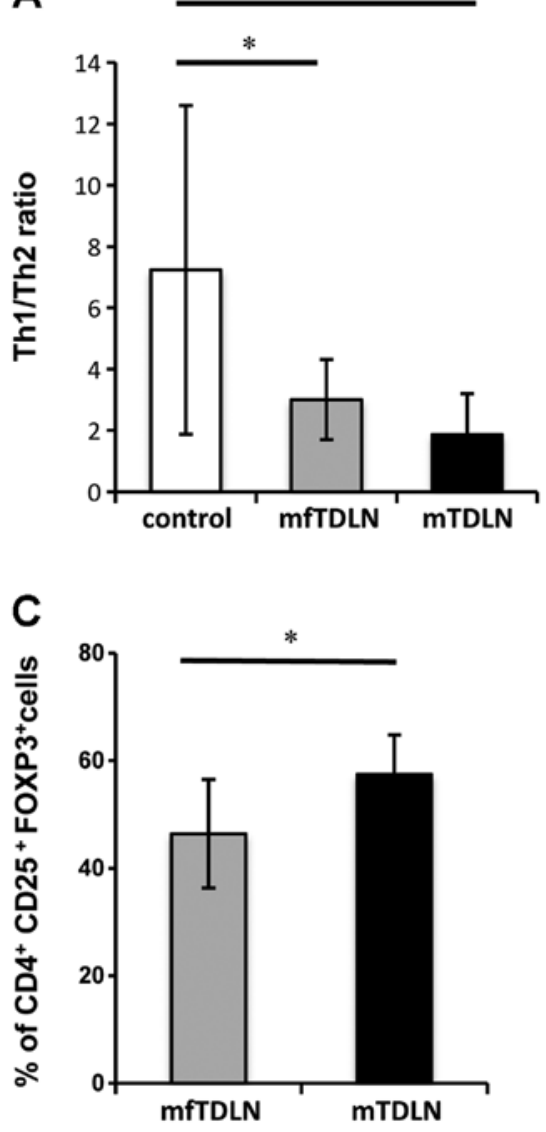

E

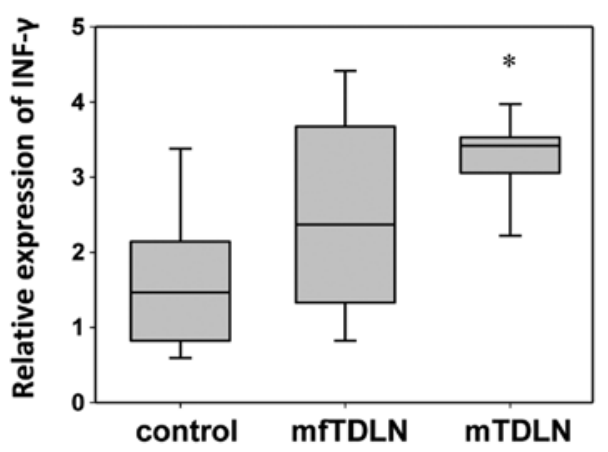

B

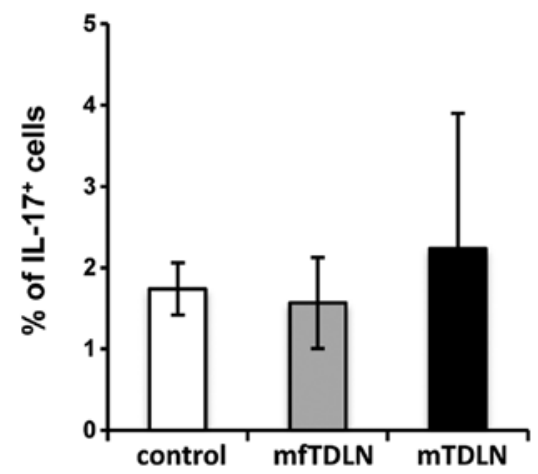

D

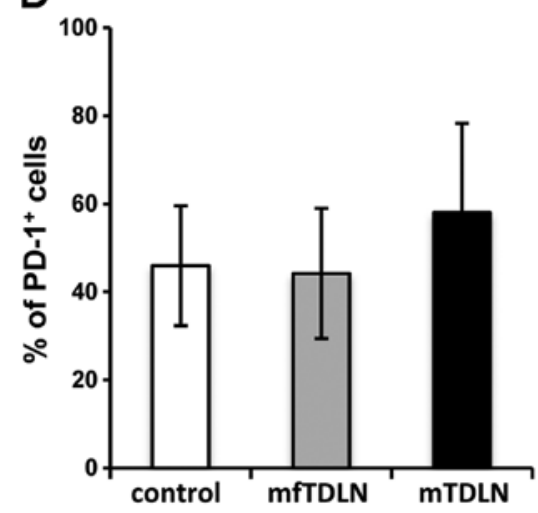

$\mathbf{F}$

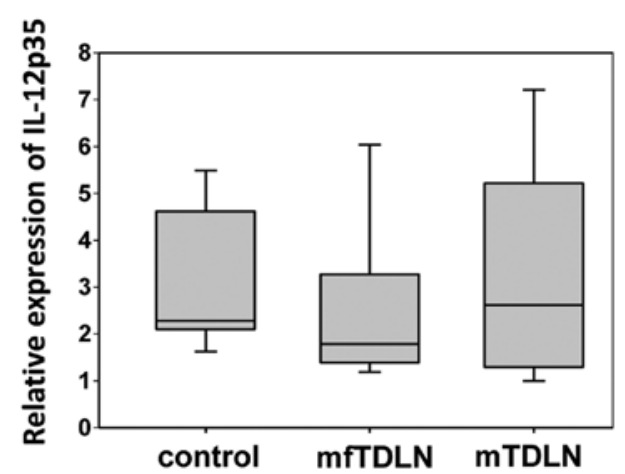

Figure 2. $\mathrm{CD}^{+}$effector $\mathrm{T}$ cell subsets and $\mathrm{T}$ cell exhaustion in TDLN. Isolated $\mathrm{CD} 4^{+} \mathrm{T}$ cells were analyzed by intracellular cytokine staining using multicolor flow cytometry. (A) Th1 and Th2 cells were detected by intracellular INF- $\gamma$ and IL-4 staining, respectively. The Th1/Th2 ratio was assessed by analyzing the proportion of INF- $\gamma^{+}$and IL- $4^{+}$cells among total CD $4^{+}$cells. Significant differences in the Th1/Th2 ratio were detected between mfTDLN and mTDLN, and between the control lymph nodes and mfTDLN. (B) Th17 cells were detected by intracellular IL-17 staining, and the proportion of IL-17 $7^{+}$among total $\mathrm{CD} 4^{+}$cells is shown. No significant differences in the proportion of Th17 cells were detected among the three groups. (C) Treg cells were detected based on the surface expression of $\mathrm{CD}^{+} \mathrm{CD} 25^{+}$and intracellular FOXP3 staining, and the proportion of FOXP3 $3^{+}$cells among total $\mathrm{CD} 4{ }^{+} \mathrm{CD} 25^{+}$cells is shown. A significant difference between mfTDLN and mTDLN was detected. (D) To assess the exhaustion of CD4 $4^{+} \mathrm{T}$ cells, PD-1 expression on CD4 $4^{+} \mathrm{T}$ cells was analyzed by flow cytometry. There is no significant differences in PD- $1^{+} \mathrm{CD} 4^{+}$cell infiltration among three groups. Relative mRNA expression of (E) IFN- $\gamma$ and (F) IL-12p35 was compared among control TDLN, mfTDLN and mTDLN by qRT-PCR. Significantly higher IFN- $\gamma$ expression was observed in control TDLN compared to mfTDLN $(\mathrm{P}=0.012)$. An asterisk (*) indicates a significant difference $(\mathrm{P}<0.05)$. TDLN, tumor-draining lymph node; mfTDLN, metastatic-free TDLN; mTDLN, metastatic TDLN; PD-1, programmed cell death-1.

mfTDLN $(\mathrm{P}=0.015)$ and mTDLN $(\mathrm{P}=0.001)$ compared with normal lymph nodes. Although no significant differences in the Th1/Th2 ratio were found between mfTDLN and mTDLN, mTDLN and mfTDLN (Fig. 2A). The proportion of Th17 cells did not markedly differ among the three lymph node groups (Fig. 2B), whereas Treg cells were increased in mTDLN compared with mfTDLN (Fig. 2C). In addition, the number of PD- $1^{+} \mathrm{CD} 4 \mathrm{~T}$ cells appeared to be increased in mTDLN compared to mfTDLN $(\mathrm{P}=0.07)$ and normal lymph nodes $(\mathrm{P}=0.09)$, although the difference was not significant (Fig. 2D).

To examine the influence of the tumor microenvironment on the levels of the different $\mathrm{T}$ cell subsets, the mRNA expression of IFN- $\gamma$ and IL-12 was measured in lymph nodes. 
A

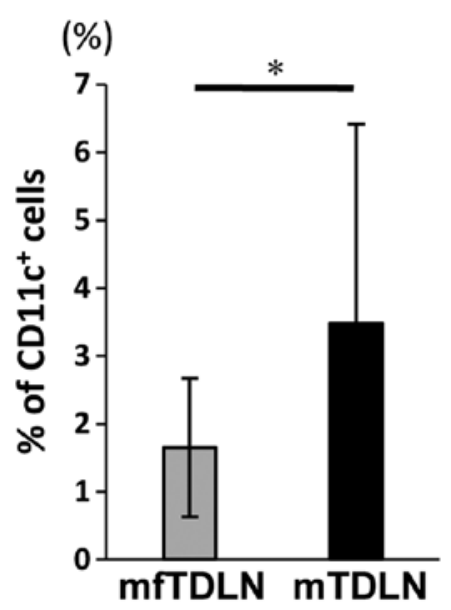

B

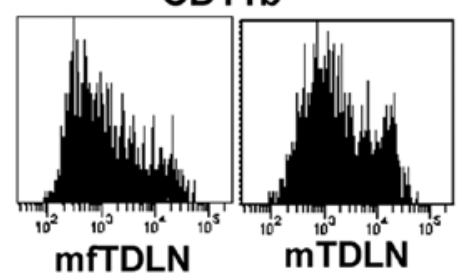

HLA-DR

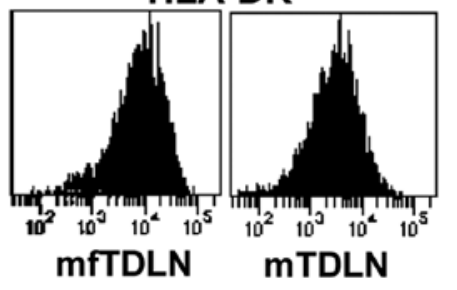

PD-L1

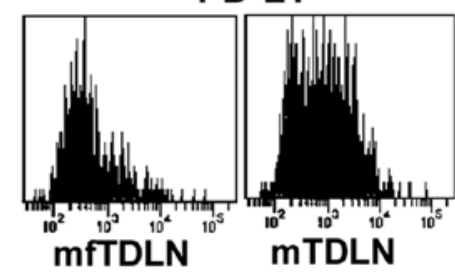

CD83

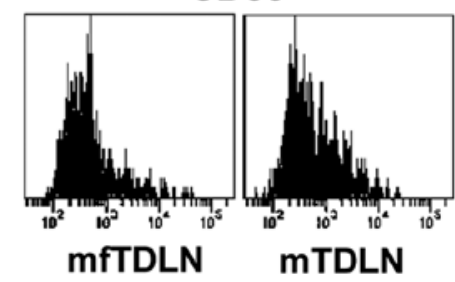

Figure 3. Phenotypic analysis and involvement of CD11 ${ }^{+}$APCs in TDLN. CD11c expression in TDLN was analyzed by flow cytometry. (A) Comparison of CD11c expression between mfTDLN and mTDLN revealed significantly higher CD11 ${ }^{+}$cell infiltration in mTDLN. (B) Analysis of APC cell-surface expression using anti-CD11b, PD-L1, HLA-DR and CD83 antibodies. CD11b and PD-L1 expressions in CD11c ${ }^{+}$cells in mTDLN were higher than those in mfTDLN. We did not observe significant differentiation in CD83 and HLA-DR. TDLN, tumor-draining lymph node; mfTDLN, metastatic-free TDLN; mTDLN, metastatic TDLN.

Although IL-12 expression was not upregulated in mTDLNs, IFN- $\gamma$ was significantly elevated $(\mathrm{P}=0.012$, Fig. $2 \mathrm{E}$ and $\mathrm{F})$.

Alteration of APC phenotype in TDLN. To determine the factors that influence $\mathrm{T}_{\mathrm{E}}$ in TDLN, the phenotype of CD11c ${ }^{+}$ cells isolated from lymph nodes was examined (Fig. 3). The

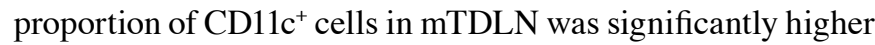
than in mfTDLN (Fig. 3A). We have previously reported that gastric cancer cells augment CD11b and PD-L2 expression

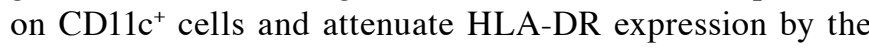
production of immunosuppressive cytokines secreted by gastric cancer cells (16). In the present study, the expression of CD11b within CD11c cells in mTDLN was higher than that in mfTDLN (Fig. 3B). PD-L1 expression on CD11c cells in mTDLN was also higher than that in mfTDLN (Fig. 3B). However, no significant differences in the expression of MHC-class II (HLA-DR) or CD83 were detected (Fig. 3B).

Neutrophil-related cytokines and proteins in TDLN. To examine whether cytokines produced by neutrophils were associated with the decrease of $\mathrm{T}_{\mathrm{E}}$ in TDLN, we determined and compared the proportion of neutrophils $\left(\mathrm{CD} 16 \mathrm{~b}^{+}\right)$in mTDLN and mfTDLN (Fig. 4A). The flow cytometric analysis revealed that neutrophil infiltration was significantly higher in mTDLN compared to mfTDLN ( $\mathrm{P}=0.016)$. Based on this finding, we next measured the mRNA expression levels of neutrophil-related chemokines in cells isolated from mTDLN and mfTDLN by qRT-PCR. Increased expression of IL-8 and/or its receptors is a characteristic of cancer and endothelial cells, infiltrating neutrophils and tumor-associated macrophages, suggesting that IL-8 functions as a significant regulatory factor within the tumor microenvironment $(22,23)$. In the present analysis, IL-8, which induces neutrophils to migrate out of the bloodstream, was expressed at significantly higher levels in mTDLNs compared to mfTDLNs $(\mathrm{P}=0.013$; Fig. 4B). Tumor-associated neutrophils reportedly contribute to tumor angiogenesis and invasion by the production of
MMP2 and MMP9 $(23,24)$, which subsequently break down extracellular matrix proteins, such as collagens and release the cryptic information. We examined mRNA expression of MMP2 and MMP9, which are required for the initial recruitment of neutrophils into tissues, and found that MMP2 but not MMP9, showed significantly higher expression in mTDLN than in mfTDLN ( $\mathrm{P}=0.013$, Fig. 4C and $\mathrm{D})$.

\section{Discussion}

In the present study, we demonstrated that the populations of effector and effector memory $\mathrm{CD}^{+} \mathrm{T}$ cells are decreased in metastatic lymph nodes, whereas Tregs, CD11b ${ }^{+}$APCs and neutrophils are increased. To the best of our knowledge, this is the first study to elucidate the changes that occur in the populations of immune cells in TDNL from human clinical samples. Considering the important roles that $\mathrm{CD}^{+} \mathrm{T}$ cells play in antitumor immune responses, these findings will help define the cellular events that occur within the tumor microenvironment in regional lymph nodes of gastric cancer.

Tumor-infiltrating CD4 ${ }^{+} \mathrm{T}_{\mathrm{EM}}$ cells capable of homing to the tumor microenvironment are responsible for longterm protection against tumors $(4,25)$. Several investigators have shown that infiltrating $\mathrm{CD}^{4} 5 \mathrm{RO}^{+}$memory $\mathrm{T}$ cells are decreased in primary tumors compared with normal tissue, and that the high tumor infiltration of such cells correlates with favorable prognosis $(7,26,27)$. In the present study, we observed that $\mathrm{T}_{\mathrm{EM}}$ are decreased in mTDLNs. As shown in a previous study, PD-L1 expressed on cancer cells has negative regulatory effects on $\mathrm{CD}^{+}$memory $\mathrm{T}$ cells (28). Thus, it is possible that $\mathrm{CD}^{+} \mathrm{T}_{\mathrm{EM}}$ in lymph nodes may generate less cytokines, such as IL-7 and IL-15 by surrounding cancer cells, resulting in a decrease of $\mathrm{T}_{\mathrm{E}}(29)$. Although it was previously reported that $\mathrm{T}_{\mathrm{CM}}$ cells correlate with antitumor functions (30), in the resent study, no significantly differences in the proportion of $\mathrm{T}_{\mathrm{CM}}$ were observed among the three types of lymph node tissue. $\mathrm{T}_{\mathrm{CM}}$ do not produce any prototypic cytokines of 
A

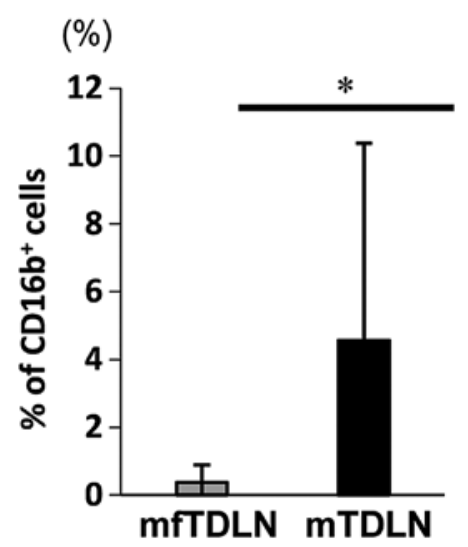

C

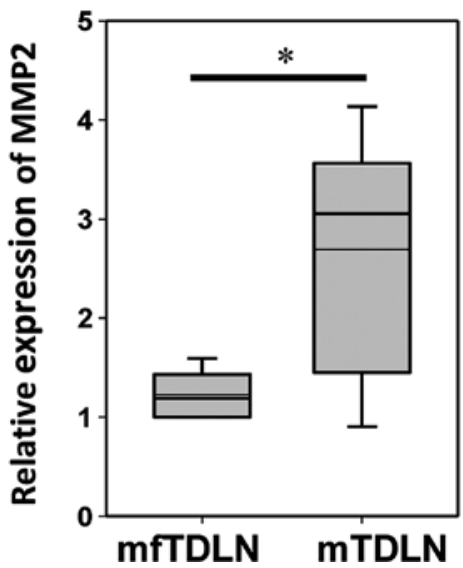

B

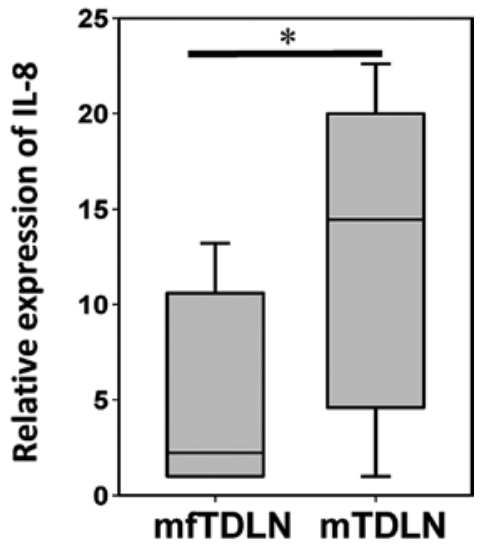

D

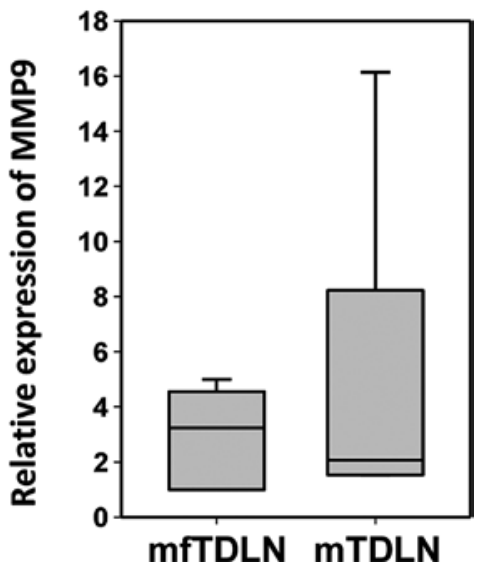

Figure 4. Neutrophil involvement and expression of neutrophil-related genes in TDLN. TDLN was mechanically dissociated and analyzed by flow cytometry

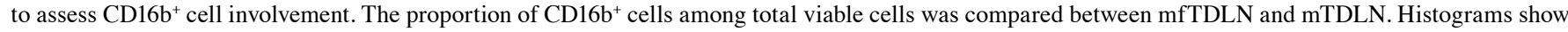
mean values \pm SD from the analysis performed for 15 mfTDLN (gray bars) and 15 mTDLN (black bars). Box and whisper plots showing the expression of neutrophil-related genes in mfTDLN and mTDLN. (A) Significantly high proportion of neutrophils in mTDLN than mfTDLN was observed. Relative mRNA expression of (B) IL-8, (C) MMP2 and (D) MMP9 was compared between mfTDLN and mTDLN. Significantly higher expression of IL-8 and MMP2 was observed in mTDLN compared to mfTDLN. An asterisk (*) indicates a significant difference (P<0.05). TDLN, tumor-draining lymph node; mfTDLN, metastatic-free TDLN; mTDLN, metastatic TDLN; MMP2, matrix metalloproteinase 2; MMP9, matrix metalloproteinase 9.

effector cells immediately after stimulation $(31,32)$, suggesting that $\mathrm{T}_{\mathrm{CM}}$ cells likely undergo a secondary response. However, since large differences in the proportion of $\mathrm{T}_{\mathrm{CM}}$ were observed between patients in the present study, future studies examining a greater number of patients may be required to better elucidate the contribution of $\mathrm{T}_{\mathrm{CM}}$ towards long-term antitumor responses.

$\mathrm{T}_{\mathrm{EM}}$ produce signals that are involved in the generation of $T_{E}$ cells. In the present study, we found that the population of $\mathrm{T}_{\mathrm{E}}$ is increased in mfTDLN compared with control TDLN, suggesting the existence of an intratumoral adaptive immune response. Alternatively, it is possible that subsets of Tregs or Th2 cells that regulate antitumor immune responses are augmented in mfTDLN. CD4 ${ }^{+} \mathrm{T}_{\mathrm{E}}$ cells can change their cytokine secretion profiles by genetic reprogramming $(33,34)$. Our present data showed that the Th1/Th2 ratio is decreased in mTDLN, this finding is consistent with the decrease in the $\mathrm{T}_{\mathrm{EM}}$ population in mTDLN. Although our results also demonstrated that the population of Treg cells is increased in mTDLN, we did not find direct evidence for the link between increasing numbers of Tregs and metastasis. However, our finding that IFN- $\gamma$ expression is upregulated in mTDLN may be related with the protumoral functions of IFN- $\gamma$, including the differentiation of Tregs and suppression of cytotoxic T lymphocytes $(35,36)$.

The underlying cellular mechanism of local immune regulation in regional lymph nodes remains to be determined. We speculate that naïve $\mathrm{CD} 4^{+} \mathrm{T}$ cells which attach to regulatory dendritic cells (DC) fail to receive further stimulatory signals. These DCs, in turn, may be affected by immunosuppressive cytokines, such as transforming growth factor (TGF)- $\beta$ and IL-10 produced by cancer cells. We previously reported that

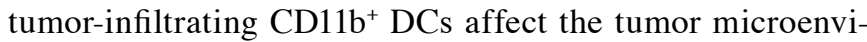
ronment and prognosis (16), and that a positive correlation exists between the number of tumor-infiltrating CD11b ${ }^{+}$APC and Treg cells. In the present study, an increased population of CD11 $\mathrm{c}^{+}$APCs, which augment expression of the negative stimulatory markers CD11b and PD-L1 was observed in mTDLN from the gastric cancer patients.

Moreover, in the present study, DCs in mTDLN did not show downregulated expression of MHC class II (HLA-DR) and did not upregulate CD83 expression, suggesting that they had a semi-mature phenotype. These findings, taken together with our present results, suggest that gastric cancer 
cells promotes the differentiation of APC into tolerogenic semi-mature CD11b ${ }^{+}$PD-L1 ${ }^{+}$APC, which induce the proliferation of Tregs, resulting in decreased populations of $\mathrm{T}_{\mathrm{E}}$ and $\mathrm{T}_{\mathrm{EM}}$.

Tumor-infiltrating neutrophils consist of antitumoral (N1) and pro-tumoral (N2) types, which have contrasting roles in cancer immunity (37). Neutrophils also have the ability to inhibit the antitumoral function of DC, and neutrophil-derived ectosomes, cathelicidins and elastase suppress DC activation and maturation (38). Our present results indicate that N2-type neutrophils induced by gastric cancer cells also promote the formation of immunosuppressive DC and correlate with the skewed $\mathrm{CD}^{+} \mathrm{T}$ cell and $\mathrm{CD} 4 \mathrm{~T}_{\mathrm{E}}$ subsets in the advanced stages of disease. In addition, we showed that IL-8 and MMP2 expression is increased in mTDLNs. Although both cancer and immune cells are able to produce IL-8 and MMP2, we could not determine which cell type predominantly produced IL-8 and MMP2 in TDLN. It has been reported that neutrophils activated by IL- 8 inhibits T cell receptor expression and cytokine secretion depending on the $\mathrm{H}_{2} \mathrm{O}_{2}$ concentration (39).

Our data suggest that MMP2 from cancer cells or neutrophils contributes to the destruction of lymphatic vessels in lymph nodes and promotes lymph node metastasis.

Several limitations of the present study warrant mention. First, we did not provide direct evidence that DCs in lymph nodes induce the proliferation of Tregs and reduce Th1 $\mathrm{CD}^{+} \mathrm{T}$ cells. Second, the association between increasing neutrophils and decreasing $\mathrm{T}_{\mathrm{E}}$ cells was not conclusively determined. Finally, the cell type responsible for the secretion of IL-8 and MMP2 was not determined. To elucidate the cellular mechanisms underlying the regulation of antitumor immune response in TDLNs, separate functional analyses of each subset of T cells, neutrophils and DCs are needed. Moreover, the molecular mechanisms of this process remain unclear, but may involve a suppressor of cytokine signal (SOCS) that regulates the proliferation of $\mathrm{T}_{\mathrm{E}}$ or $\mathrm{T}_{\mathrm{EM}}(40)$.

In conclusion, the present data indicate that $\mathrm{CD}^{+} \mathrm{T}$ cell and $T_{E}$ subsets in TDLN of gastric cancer patients are significantly affected by metastasis. Immunosuppressive cells, such

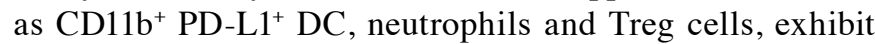
increased migration to TDLN, in which a subset of CD4 ${ }^{+}$ effector cells is skewed towards immune tolerance in the tumor microenvironment. Our findings of the local alteration of immune cells in TDLN shed light on the immunological mechanisms that occur in the tumor microenvironment and are expected to aid in the design of effective immune therapy for patients with gastric cancer.

\section{Acknowledgements}

This study was supported by a Grant-in-Aid for Scientific Research (to H.T. and K.H.).

\section{References}

1. Shurin GV, Ma Y and Shurin MR: Immunosuppressive mechanisms of regulatory dendritic cells in cancer. Cancer Microenviron 6: 159-167, 2013.

2. Sallusto F, Lenig D, Förster R, Lipp M and Lanzavecchia A: Two subsets of memory $\mathrm{T}$ lymphocytes with distinct homing potentials and effector functions. Nature 401: 708-712, 1999.
3. Sallusto $\mathrm{F}$ and Lanzavecchia A: Heterogeneity of $\mathrm{CD} 4^{+}$memory $\mathrm{T}$ cells: Functional modules for tailored immunity. Eur $\mathrm{J}$ Immunol 39: 2076-2082, 2009.

4. Battaglia A, Buzzonetti A, Baranello C, Ferrandina G, Martinelli E, Fanfani F, Scambia G and Fattorossi A: Metastatic tumour cells favour the generation of a tolerogenic milieu in tumour draining lymph node in patients with early cervical cancer. Cancer Immunol Immunother 58: 1363-1373, 2009.

5. Ito N, Suzuki Y, Taniguchi Y, Ishiguro K, Nakamura $H$ and Ohgi S: Prognostic significance of $\mathrm{T}$ helper 1 and 2 and T cytotoxic 1 and 2 cells in patients with non-small cell lung cancer. Anticancer Res 25: 2027-2031, 2005.

6. Tosolini M, Kirilovsky A, Mlecnik B, Fredriksen T, Mauger S, Bindea G, Berger A, Bruneval P, Fridman WH, Pagès F, et al: Clinical impact of different classes of infiltrating T cytotoxic and helper cells (Th1, th2, treg, th17) in patients with colorectal cancer. Cancer Res 71: 1263-1271, 2011.

7. Enomoto K, Sho M, Wakatsuki K, Takayama T, Matsumoto S, Nakamura S, Akahori T, Tanaka T, Migita K, Ito M, et al: Prognostic importance of tumour-infiltrating memory $\mathrm{T}$ cells in oesophageal squamous cell carcinoma. Clin Exp Immunol 168: 186-191, 2012.

8. Kristensen VN, Vaske CJ, Ursini-Siegel J, Van Loo P, Nordgard SH, Sachidanandam R, Sørlie T, Wärnberg F, Haakensen VD, Helland $\AA$, et al: Integrated molecular profiles of invasive breast tumors and ductal carcinoma in situ (DCIS) reveal differential vascular and interleukin signaling. Proc Natl Acad Sci USA 109: 2802-2807, 2012.

9. Bates GJ, Fox SB, Han C, Leek RD, Garcia JF, Harris AL and Banham AH: Quantification of regulatory T cells enables the identification of high-risk breast cancer patients and those at risk of late relapse. J Clin Oncol 24: 5373-5380, 2006.

10. Gnerlich JL, Mitchem JB, Weir JS, Sankpal NV, Kashiwagi H, Belt BA, Porembka MR, Herndon JM, Eberlein TJ, Goedegebuure P, et al: Induction of Th17 cells in the tumor microenvironment improves survival in a murine model of pancreatic cancer. J Immunol 185: 4063-4071, 2010.

11. Mougiakakos D, Johansson CC, Trocme E, All-Ericsson C, Economou MA, Larsson O, Seregard S and Kiessling R: Intratumoral forkhead box $\mathrm{P} 3$-positive regulatory $\mathrm{T}$ cells predict poor survival in cyclooxygenase-2-positive uveal melanoma. Cancer 116: 2224-2233, 2010.

12. Lee HE, Park DJ, Kim WH, Kim HH and Lee HS: High FOXP3 ${ }^{+}$ regulatory T-cell density in the sentinel lymph node is associated with downstream non-sentinel lymph-node metastasis in gastric cancer. Br J Cancer 105: 413-419, 2011.

13. Kim M, Grimmig T, Grimm M, Lazariotou M, Meier E, Rosenwald A, Tsaur I, Blaheta R, Heemann U, Germer CT, et al: Expression of Foxp3 in colorectal cancer but not in Treg cells correlates with disease progression in patients with colorectal cancer. PLoS One 8: e53630, 2013.

14. Nakamura R, Sakakibara M, Nagashima T, Sangai T, Arai M, Fujimori T, Takano S, Shida T, Nakatani Y and Miyazaki M: Accumulation of regulatory $\mathrm{T}$ cells in sentinel lymph nodes is a prognostic predictor in patients with node-negative breast cancer. Eur J Cancer 45: 2123-2131, 2009.

15. Deng L, Zhang H, Luan Y, Zhang J, Xing Q, Dong S, Wu X, Liu M and Wang S: Accumulation of foxp $3^{+} \mathrm{T}$ regulatory cells in draining lymph nodes correlates with disease progression and immune suppression in colorectal cancer patients. Clin Cancer Res 16: 4105-4112, 2010.

16. Okita Y, Tanaka H, Ohira M, Muguruma K, Kubo N, Watanabe M, Fukushima W and Hirakawa K: Role of tumorinfiltrating CD11 $\mathrm{b}^{+}$antigen-presenting cells in the progression of gastric cancer. J Surg Res 186: 192-200, 2014.

17. Carus A, Ladekarl M, Hager H, Nedergaard BS and Donskov F: Tumour-associated $\mathrm{CD} 66 \mathrm{~b}^{+}$neutrophil count is an independent prognostic factor for recurrence in localised cervical cancer. Br J Cancer 108: 2116-2122, 2013.

18. Zhao JJ, Pan K, Wang W, Chen JG, Wu YH, Lv L, Li JJ, Chen YB, Wang DD, Pan QZ, et al: The prognostic value of tumor-infiltrating neutrophils in gastric adenocarcinoma after resection. PLoS One 7: e33655, 2012.

19. Houghton AM: The paradox of tumor-associated neutrophils: Fueling tumor growth with cytotoxic substances. Cell Cycle 9: 1732-1737, 2010.

20. Japanese Gastric Cancer Association: Japanese classification of gastric carcinoma: 3rd English edition. Gastric Cancer 14: 101-112, 2011. 
21. Sano T and Aiko T: New Japanese classifications and treatment guidelines for gastric cancer: Revision concepts and major revised points. Gastric Cancer 14: 97-100, 2011.

22. Waugh DJ and Wilson C: The interleukin-8 pathway in cancer. Clin Cancer Res 14: 6735-6741, 2008.

23. Shamamian P, Schwartz JD, Pocock BJ, Monea S, Whiting D, Marcus SG and Mignatti P: Activation of progelatinase A (MMP-2) by neutrophil elastase, cathepsin G, and proteinase-3: A role for inflammatory cells in tumor invasion and angiogenesis. J Cell Physiol 189: 197-206, 2001.

24. Bekes EM, Schweighofer B, Kupriyanova TA, Zajac E, Ardi VC, Quigley JP and Deryugina EI: Tumor-recruited neutrophils and neutrophil TIMP-free MMP-9 regulate coordinately the levels of tumor angiogenesis and efficiency of malignant cell intravasation. Am J Pathol 179: 1455-1470, 2011.

25. Poschke I, De Boniface J, Mao Y and Kiessling R: Tumor-induced changes in the phenotype of blood-derived and tumor-associated $\mathrm{T}$ cells of early stage breast cancer patients. Int J Cancer 131: 1611-1620, 2012.

26. Pagès F, Kirilovsky A, Mlecnik B, Asslaber M, Tosolini M Bindea G, Lagorce C, Wind P, Marliot F, Bruneval P, et al: In situ cytotoxic and memory $\mathrm{T}$ cells predict outcome in patients with early-stage colorectal cancer. J Clin Oncol 27: 5944-5951, 2009.

27. Mlecnik B, Tosolini M, Kirilovsky A, Berger A, Bindea G, Meatchi T, Bruneval P, Trajanoski Z, Fridman WH, Pagès F, et al: Histopathologic-based prognostic factors of colorectal cancers are associated with the state of the local immune reaction. J Clin Oncol 29: 610-618, 2011.

28. Dong H, Strome SE, Salomao DR, Tamura H, Hirano F, Flies DB, Roche PC, Lu J, Zhu G, Tamada K, et al: Tumor-associated B7-H1 promotes T-cell apoptosis: A potential mechanism of immune evasion. Nat Med 8: 793-800, 2002.

29. Bradley LM, Haynes L and Swain SL: IL-7: Maintaining T-cell memory and achieving homeostasis. Trends Immunol 26 172-176, 2005.
30. Wu F, Zhang W, Shao H, Bo H, Shen H, Li J, Liu Y, Wang T, Ma W and Huang S: Human effector T cells derived from central memory cells rather than CD8 ${ }^{+} \mathrm{T}$ cells modified by tumor-specific TCR gene transfer possess superior traits for adoptive immunotherapy. Cancer Lett 339: 195-207, 2013.

31. Kedzierska K, Valkenburg SA, Doherty PC, Davenport MP and Venturi V: Use it or lose it: Establishment and persistence of T cell memory. Front Immunol 3: 357, 2012.

32. Mueller SN, Gebhardt T, Carbone FR and Heath WR: Memory $\mathrm{T}$ cell subsets, migration patterns, and tissue residence. Annu Rev Immunol 31: 137-161, 2013.

33. Zhou L, Chong MM and Littman DR: Plasticity of $\mathrm{CD}^{+}{ }^{+} \mathrm{T}$ cell lineage differentiation. Immunity 30: 646-655, 2009.

34. Zhu J and Paul WE: Heterogeneity and plasticity of $\mathrm{T}$ helper cells. Cell Res 20: 4-12, 2010.

35. Brody JR, Costantino CL, Berger AC, Sato T, Lisanti MP, Yeo CJ, Emmons RV and Witkiewicz AK: Expression of indoleamine 2,3-dioxygenase in metastatic malignant melanoma recruits regulatory $\mathrm{T}$ cells to avoid immune detection and affects survival. Cell Cycle 8: 1930-1934, 2009.

36. Ostrand-Rosenberg S and Sinha P: Myeloid-derived suppressor cells: Linking inflammation and cancer. J Immunol 182: 4499-4506, 2009.

37. Fridlender ZG, Sun J, Kim S, Kapoor V, Cheng G, Ling L, Worthen GS and Albelda SM: Polarization of tumor-associated neutrophil phenotype by TGF-beta: 'N1' versus 'N2' TAN. Cancer Cell 16: 183-194, 2009.

38. Schuster S, Hurrell B and Tacchini-Cottier F: Crosstalk between neutrophils and dendritic cells: A context-dependent process. J Leukoc Biol 94: 671-675, 2013

39. Schmielau J and Finn OJ: Activated granulocytes and granulocyte-derived hydrogen peroxide are the underlying mechanism of suppression of $\mathrm{t}$-cell function in advanced cancer patients. Cancer Res 61: 4756-4760, 2001.

40. Palmer DC and Restifo NP: Suppressors of cytokine signaling (SOCS) in T cell differentiation, maturation, and function. Trends Immunol 30: 592-602, 2009. 